\title{
Synchronization of Neuronal Circuits with Ring Connection on PSpice
}

\author{
Lihua Yuan, Guodong Ren, and Chunni Wang \\ Department of Physics, Lanzhou University of Technology, Lanzhou 730050, China \\ Correspondence should be addressed to Chunni Wang; wangcn05@163.com
}

Received 18 December 2015; Accepted 18 February 2016

Academic Editor: M. Junaid Khan

Copyright ( 2016 Lihua Yuan et al. This is an open access article distributed under the Creative Commons Attribution License, which permits unrestricted use, distribution, and reproduction in any medium, provided the original work is properly cited.

\begin{abstract}
The modes of electric activities in neurons and wave propagation between neurons can be changed by using appropriate external forcing or coupling type. Based on the PSpice tool, the coupling synchronization between three Hindmarsh-Rose neurons is investigated. The neuronal circuit is also designed, and the Schmitt trigger circuit is used as peak detector by transmitting the analog signal into digital signal; thus, the rhythm of electric activities of neurons can be analyzed. By selecting different initial states (spiking or bursting), different synchronization states are found. In the case of spiking state, appropriate coupling intensity is effective in realizing rhythm synchronization and then homogenous state is observed by further increasing the coupling intensity. In the case of bursting state, rhythm synchronization is enhanced under appropriate coupling intensity while desynchronization occurs by further increasing the coupling intensity.
\end{abstract}

\section{Introduction}

Neuron is the basic unit in neuronal system, and the electric activity can be described by some neuron modes [1-3]. In fact, signals can be transmitted among neurons of networks composed of a large number of neurons under multifarious types of coupling. Neuronal networks with different types of topologies can be proposed to detect the signal or wave propagation and the transition of collective electric activities in network of neurons. Based on the proposed neuron models, the dynamical properties of single neuron, collective behaviors of neurons, and transition of neuronal electric activities have been investigated extensively [4-7]. For example, synchronization between electric activities of neurons [8-10], phase synchronization $[11,12]$, coherence resonance [13-18], transition of electric modes in neurons [19-24], and the development and transition of spatial pattern in neuronal networks [25-29] have been discussed in numerical studies. In a biological experimental way, isolated neurons can be immersed into certain ionic liquid to detect the effect of external forcing from potassium, sodium, and even the leakage current [30-32]. On the other hand, it becomes interesting to design artificial neuronal circuit with low power consumption [33-36], and thus neuronal activity can be reproduced from the intelligent neuronal circuits. Furthermore, the development and transition of collective electric activities of neurons can be detected within the coupled neuronal circuits, and it can give some useful guidance to discern the collective electric activities in large scale network of neurons.

In a general way, the scheme of numerical studies could be feasible to study dynamical behaviors of mode transition of neuronal activities based on some theoretical models. However, it shows a reliable way to judge the effectiveness of control schemes based on neuronal circuit composed of analog elements by using some commercial circuit elements. There is some diversity between neurons in realistic neuronal systems, and different responses occur when identical or nonidentical external forcing is imposed on the neuron. As a result, it is more important to study the synchronization and desynchronization between rhythms of electric activities than the topic of complete synchronization. In the case of signal detection from neuronal circuit, signal converter is often used to transmit analog signal to digital signal; thus, the rhythm of signal can be exactly detected, particularly for the case of bursting state. The connection between neurons 
in realistic neuronal system is much complex, and a coupling with electric type is more effective in changing electric mode of neurons than the chemical coupling type. It is believed that neurons should be connected with small-world type connection in detecting the collective electric activities of neurons. In fact, the nearest neighbor connection is also effective in describing the coupling action between adjacent neurons when the size of network is small; for example, finite neurons are connected to design a neuronal network. In this paper, firstly, a feasible circuit is designed for HindmarshRose neuron mode [2], then three neuronal circuits are used to design a coupled neuronal network, and the rhythm synchronization of electric activity of coupled neuronal network will be investigated under mutual coupling and unidirectional coupling, respectively. Based on the PSpice tool [37], the gain in coupling controller is carefully adjusted to detect the modulation and transition of synchronization of network, it is found that synchronization can be reached between the coupled neurons, and it can offer some reliable information and tools to study the synchronization of coupled neuronal circuits.

\section{Circuits Implementation of Single Neuron and Coupled Neurons}

The Hindmarsh-Rose neuron [2, 38] can describe the main properties of neuronal activities, and the mathematical model is feasible for bifurcation analysis, and thus different electric modes can be observed. The dynamical equations can be described by

$$
\begin{aligned}
& \dot{x}=y-a x^{3}+b x^{2}-z+I_{\text {ext }} \\
& \dot{y}=c-d x^{2}-y \\
& \dot{z}=r[s(x+1.6)-z],
\end{aligned}
$$

where $\dot{x}, \dot{y}, \dot{z}$ define the membrane potential, recovery variable associated with the fast current $\left(\mathrm{Na}^{+}\right.$or $\left.\mathrm{K}^{+}\right)$, and a slow adaptation current probably linked to $\mathrm{Ca}^{2+}$, respectively. $I_{\text {ext }}$ denotes the external forcing current, parameters are often selected as $a=1, b=3, c=1, d=5, r=0.006$, and $s=4$, and the dynamical mode can create different electric modes by adjusting the external forcing carefully. Based on the PSpice tool, a practical neuronal circuit is designed in Figure 1.

To discern the rhythm of electric activity of neuron, the output voltage X_A in Figure 1 is converted into digital signal by using Schmitt trigger circuit so that the peak could be detected, which is shown in Figure 2.

According to Figure 1, different external forcing currents are imposed on the neuronal circuit and the output digital signals are plotted in Figure 3.

It is found in Figure 3 that analog signal from the single neuronal circuit can be converted into digital signal by passing through Schmitt trigger circuit, and the outputs show spiking, bursting state under appropriate external forcing. Furthermore, the collective activities of coupled neuronal circuits under nonreversing (or unidirectional) coupling will be investigated by connecting three neuronal circuits in ring type, and realistic circuit is shown in Figure 4.
Within the coupled neuronal network with three neurons being included, the local kinetics of each node is described by Hindmarsh-Rose neurons, and the dynamical equations for the coupled network are described by

$$
\begin{aligned}
& \dot{x}_{1}=y_{1}-a x_{1}^{3}+b x_{1}^{2}-z_{1}+I_{\mathrm{ext}, 1}+D_{1}\left(x_{3}-x_{1}\right) \\
& \dot{y}_{1}=c-d x_{1}^{2}-y_{1} \\
& \dot{z}_{1}=r\left[s\left(x_{1}+1.6\right)-z_{1}\right] \\
& \dot{x}_{2}=y_{2}-a x_{2}^{3}+b x_{2}^{2}-z_{2}+I_{\mathrm{ext}, 2}+D_{2}\left(x_{1}-x_{2}\right) \\
& \dot{y}_{2}=c-d x_{2}^{2}-y_{2} \\
& \dot{z}_{2}=r\left[s\left(x_{2}+1.6\right)-z_{2}\right] \\
& \dot{x}_{3}=y_{3}-a x_{3}^{3}+b x_{3}^{2}-z_{3}+I_{\mathrm{ext}, 3}+D_{3}\left(x_{2}-x_{3}\right) \\
& \dot{y}_{3}=c-d x_{3}^{2}-y_{3} \\
& \dot{z}_{3}=r\left[s\left(x_{3}+1.6\right)-z_{3}\right]
\end{aligned}
$$

where the variables and parameters are defined as mentioned above $[34,37]$, the subscript $i$ represents the node position, $I_{\text {ext }, i}$ is the external forcing current on neuron connected to node $i$, and $D_{1}, D_{2}$, and $D_{3}$ are coupling intensity between neurons; for simplicity, $D_{1}=D_{2}=D_{3}$ will be used. The synchronization problem in (2) could be investigated in detail by changing the coupling intensity and controllable parameters in numerical studies. Take Figure 4 as an example by analyzing the coupling between neurons N3 and N1 with nonreversing (or unidirectional) type, an effective subtracting circuit can be designed by using the resistances RY43, RY44, RY47, and RY49 and Operational Amplifier U13A; thus, the error of membrane potentials between two coupled neurons can be generated. The resistances RY45 and RY48 decrease the error of membrane potentials with a gain about 0.17 , the seesaw amplifier is composed of the resistance RY46, R1COUP2, and Operational Amplifier U13B, and gain for Operational Amplifier is described by R1COUP2/RY46; as a result, the coupling intensity is $0.17 \times \mathrm{R} 1 \mathrm{COUP} 2 / \mathrm{RY} 46$. For simplicity, the same coupling intensity and external forcing currents are used for each neuron. In the case of nonreversing (or unidirectional) coupling, the external forcing current $I_{\text {ext }}=120 \mu \mathrm{A}$, the coupling intensity is selected within $0.17 \sim 1$, the circuit simulation based on PSpice shows that neuronal circuit will keep quiescent state, and then the external forcing current is increased to $I_{\text {ext }}=130 \mu \mathrm{A}$, and coupling intensity is selected by 0.17 in Figure 5; the case for coupling intensity 0.34 is shown in Figure 6.

The results in Figures 5 and 6 confirm that each neuronal circuit creates spiking state with higher external forcing current, and stronger coupling intensity is effective in enhancing rhythm synchronization of electric activities. Indeed, further increasing the coupling intensity, all neurons step into quiescent state after transient synchronization period, and the results are shown in Figure 7.

The results in Figure 7 show that the network of coupled neuronal circuits can reach synchronization by increasing 


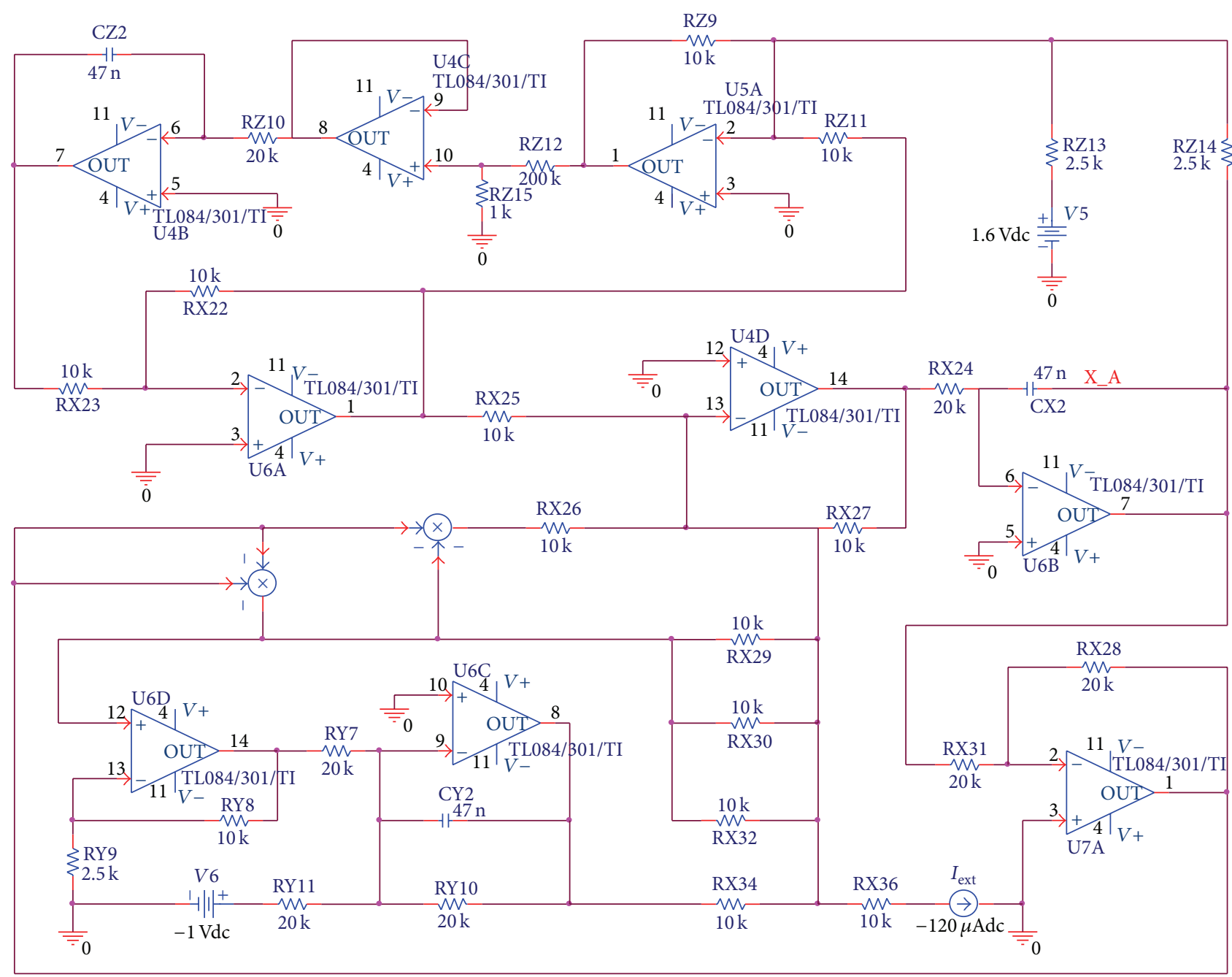

FIGURE 1: The artificial circuit for single Hindmarsh-Rose is illustrated by imposing external forcing, X_A is the output voltage, and $I_{\text {ext }}$ denotes the external forcing current.

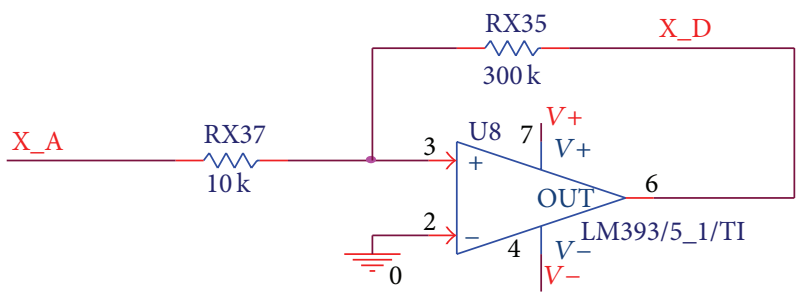

FIGURE 2: Schmitt trigger circuit: X_A is input analog signal and X_D is output digital signal.

the coupling intensity; furthermore, it develops into homogeneous state after a transient period. The potential mechanism is that each neuron is driven to step into quiescent state due to negative feedback. It is also interesting to investigate the bursting case, by selecting external forcing current $I_{\text {ext }}=$ $150 \mu \mathrm{A}$, and the experimental results are shown in Figures 8 and 9 .
It is found in Figure 8 that coupled neuronal circuits can still reach rhythm synchronization in the case of bursting state under appropriate coupling intensity.

It is found in Figure 9 that bursting synchronization can be stabilized under appropriate coupling intensity. However, transition from bursting synchronization to desynchronization can be observed by further increasing the coupling intensity, and the experimental results are shown in Figures 10 and 11 .

It is found that transition from synchronization to desynchronization is induced by selecting stronger coupling intensity in the case of bursting state; the potential mechanism could be that each neuron undergoes coupling feedback with diversity in the case of directional coupling. In a word, appropriate coupling enhances rhythm synchronization while stronger coupling intensity accounts for homogeneous state after transient spiking synchronization, and desynchronization occurs after bursting synchronization. In realistic neuronal system, bidirectional coupling could emerge between neurons, the dynamical equations are described by (2), and 


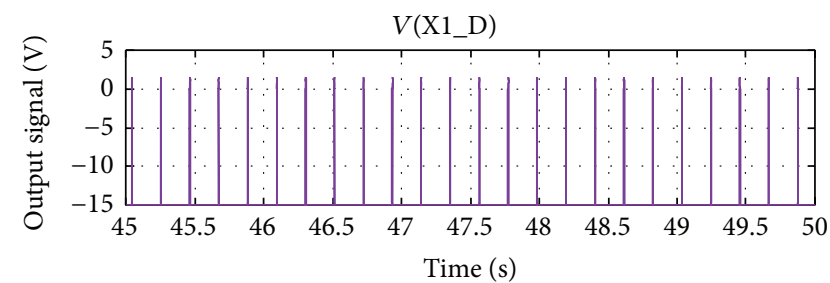

(a)

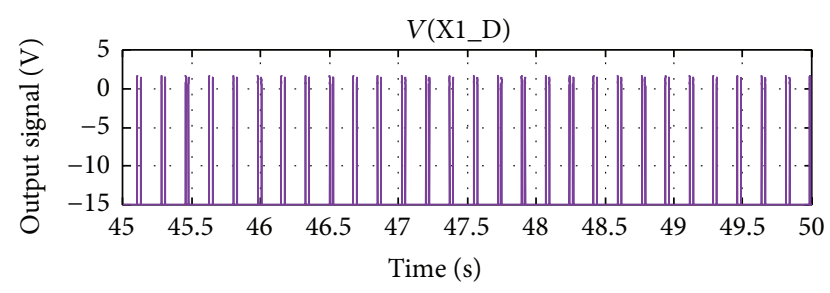

(b)

FIGURE 3: The output digital signal: the external forcing current imposed on the circuits is selected as 130 and $150 \mu \mathrm{A}(\mathrm{a}, \mathrm{b})$.

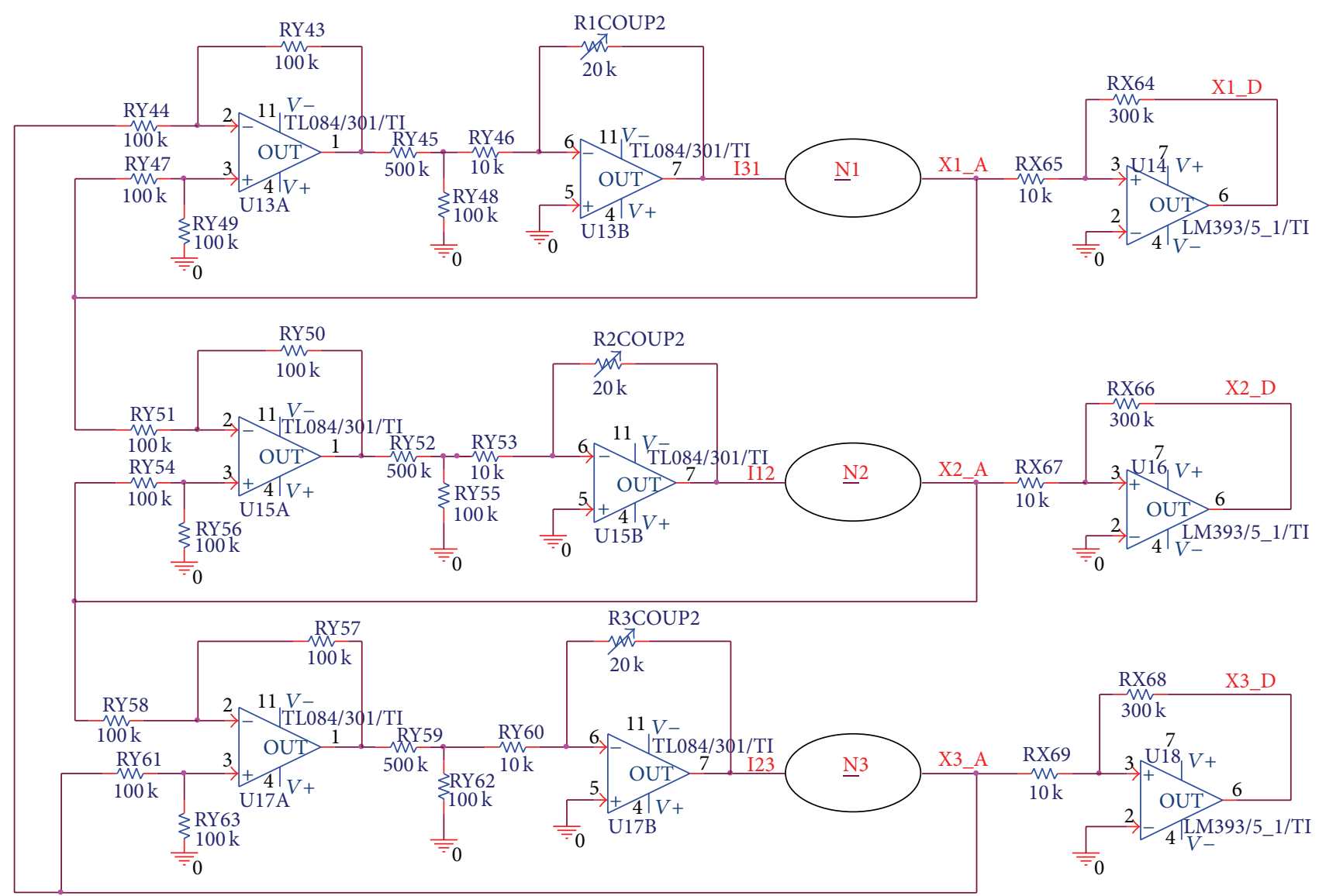

FIGURE 4: The coupled neuronal circuits for three neurons under nonreversing (or unidirectional) coupling type: X1_D, X2_D, and X3_D are the output digital signals from three different neuronal circuits and I31, I12, and I23 describe the coupling feedback term on the first, second, and third neurons, respectively.

realistic circuits between three coupled neurons based on PSpice are shown in Figure 12:

$$
\begin{aligned}
& \dot{x}_{1}=y_{1}-a x_{1}^{3}+b x_{1}^{2}-z_{1}+I_{\text {ext, } 1}+D_{1}\left(x_{3}+x_{2}-2 x_{1}\right) \\
& \dot{y}_{1}=c-d x_{1}^{2}-y_{1} \\
& \dot{z}_{1}=r\left[s\left(x_{1}+1.6\right)-z_{1}\right] \\
& \dot{x}_{2}=y_{2}-a x_{2}^{3}+b x_{2}^{2}-z_{2}+I_{\text {ext, } 2}+D_{2}\left(x_{3}+x_{1}-2 x_{2}\right) \\
& \dot{y}_{2}=c-d x_{2}^{2}-y_{2}
\end{aligned}
$$

$$
\begin{aligned}
& \dot{z}_{2}=r\left[s\left(x_{2}+1.6\right)-z_{2}\right] \\
& \dot{x}_{3}=y_{3}-a x_{3}^{3}+b x_{3}^{2}-z_{3}+I_{\mathrm{ext}, 3}+D_{3}\left(x_{2}+x_{1}-2 x_{3}\right) \\
& \dot{y}_{3}=c-d x_{3}^{2}-y_{3} \\
& \dot{z}_{3}=r\left[s\left(x_{3}+1.6\right)-z_{3}\right]
\end{aligned}
$$

where the variables and parameters are selected as the case for nonreversing (or unidirectional) coupling; $D_{1}, D_{2}$, and $D_{3}$ are coupling intensity between neurons; for simplicity, $D_{1}=D_{2}=D_{3}$ will be used. The outputs for potentials 

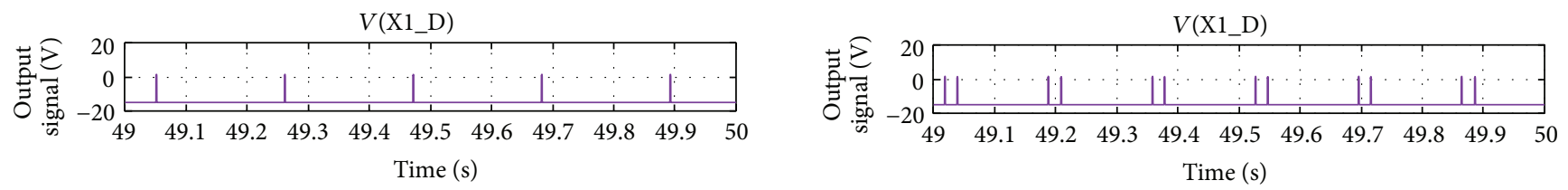

$V\left(\mathrm{X} 2 \_\mathrm{D}\right)$
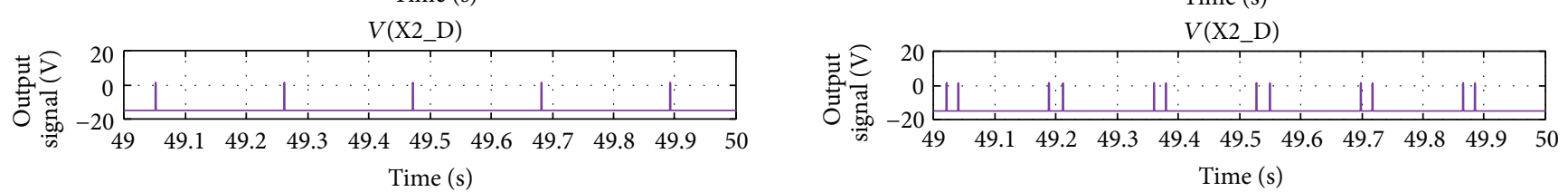

$V\left(\mathrm{X} 3 \_\mathrm{D}\right)$

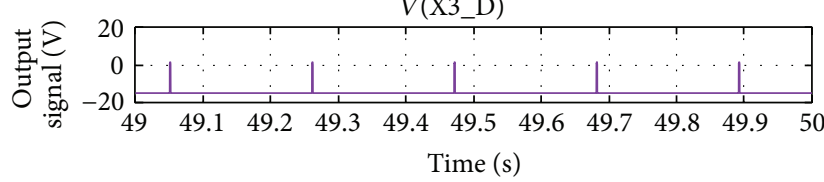

FIgURE 5: The digital outputs for three circuits via Schmitt trigger circuit: $I_{\mathrm{ext}}=130 \mu \mathrm{A}$ and coupling intensity is set as 0.17 .
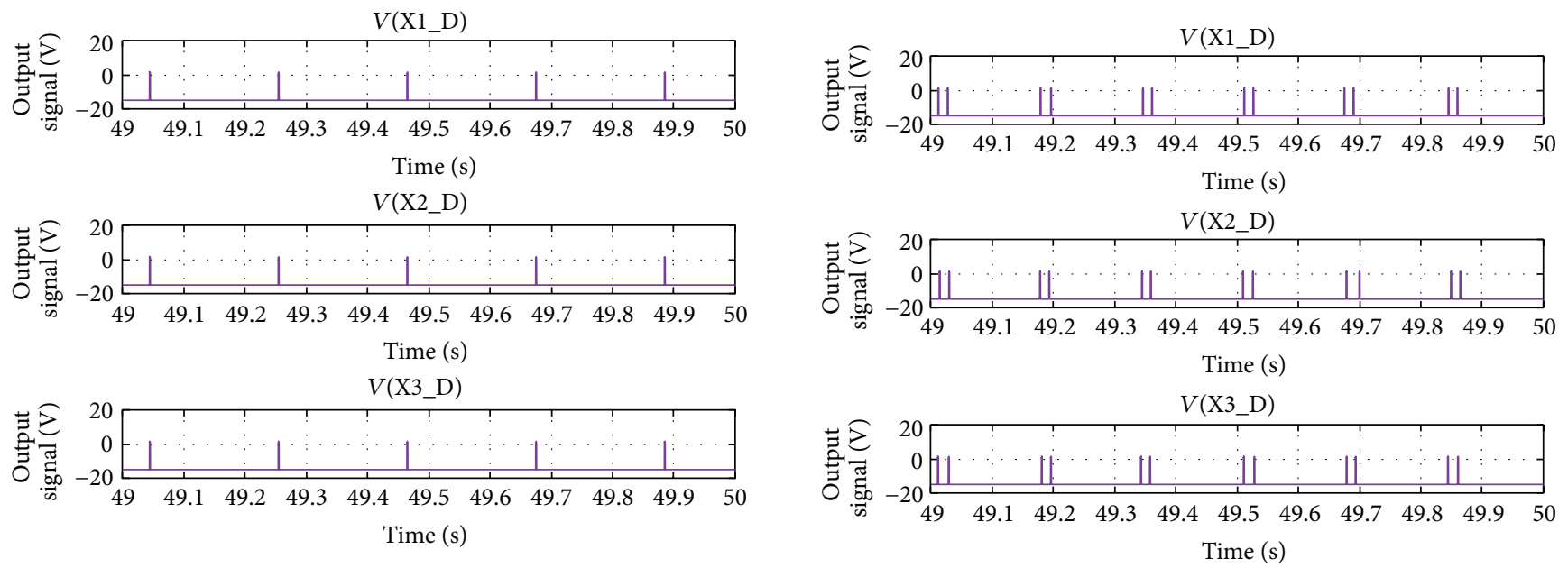

FIGURE 6: The digital outputs for three circuits via Schmitt trigger circuit: $I_{\text {ext }}=130 \mu \mathrm{A}$ and coupling intensity is set as 0.34 .
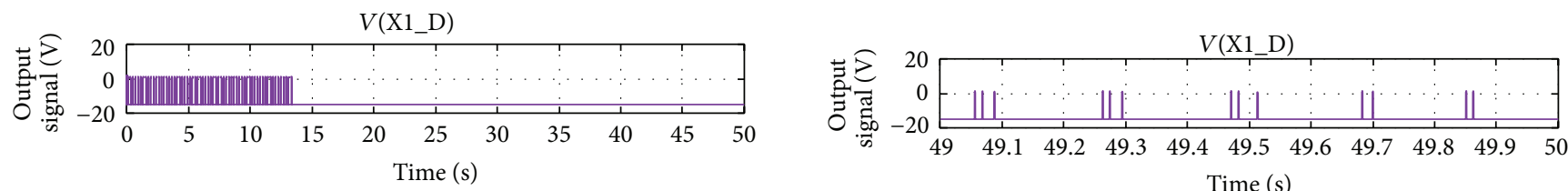

FIgURE 9: Digital outputs for three circuits via Schmitt trigger circuit: $I_{\text {ext }}=150 \mu \mathrm{A}$ and coupling intensity is set as 0.5 .
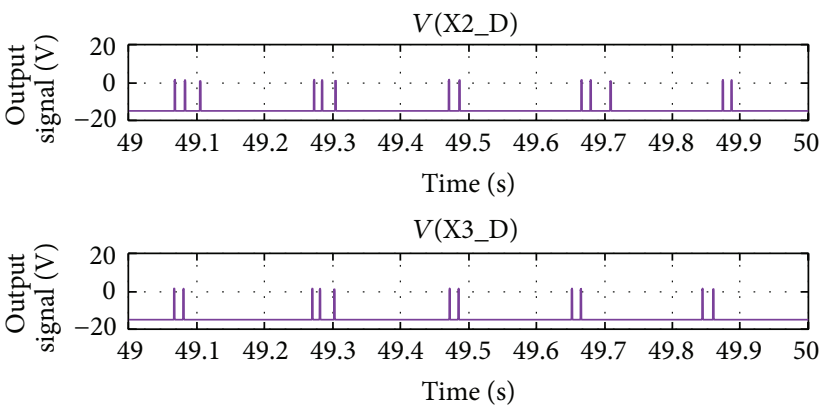

FIGURE 7: The digital outputs for three circuits via Schmitt trigger circuit: $I_{\text {ext }}=130 \mu \mathrm{A}$ and coupling intensity is set as 0.42 .

FIGURE 10: Digital outputs for three circuits via Schmitt trigger circuit: $I_{\text {ext }}=150 \mu \mathrm{A}$ and coupling intensity 1.0. 


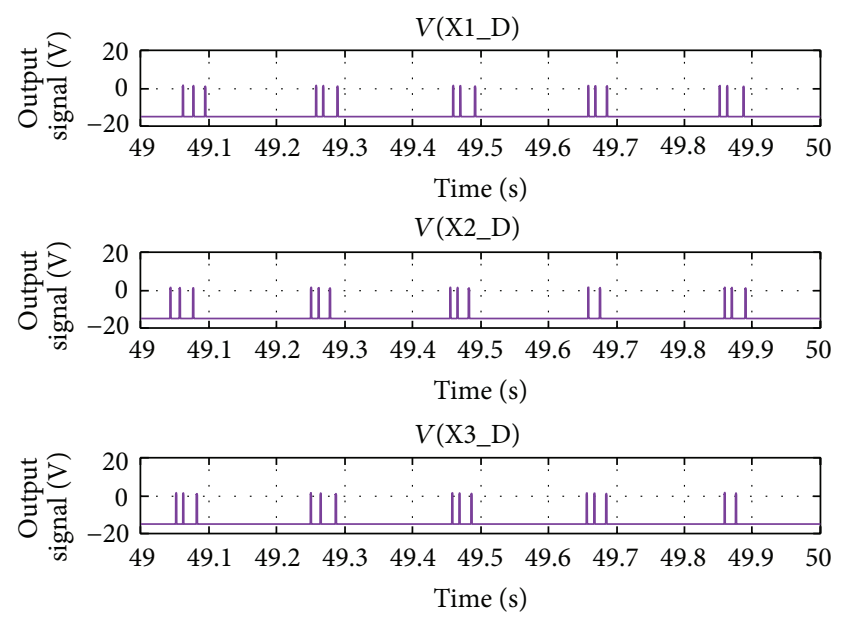

Figure 11: Digital outputs for three circuits via Schmitt trigger circuit: $I_{\text {ext }}=150 \mu \mathrm{A}$ and coupling intensity is set as 1.01 .

(voltage) are converted into digital signals via Schmitt trigger circuit, and different coupling intensities and external forcing currents are used to judge the effectiveness of the coupled neuronal circuits network. In the case of small external forcing current, for example, $I_{\text {ext }}=120 \mu \mathrm{A}$, quiescent state is observed and network shows homogeneous state. By increasing the external forcing current, neuronal circuit can produce spiking or bursting state. To observe the transition of synchronization, different coupling intensities are used to observe the outputs from the coupled neuronal circuits. At first, $I_{\mathrm{ext}}=130 \mu \mathrm{A}$, the case for spiking synchronization is investigated, and the results are plotted in Figure 13.

In Figures 13 and 14 it is found that appropriate coupling intensity is effective in realizing rhythm synchronization under bidirectional coupling type when each neuronal circuit is in spiking state; furthermore, the network of coupled neuronal circuits creates homogeneous state when each neuronal circuit of the network is decreased into quiescent state due to bidirectional coupling, and the results are similar to the case under nonreversing (or unidirectional) coupling type. However, the transition of synchronization of coupled neuronal circuits shows complexity and diversity; the output digital signals are shown in Figures 15, 16, and 17 under different forcing currents and coupling intensities.

The results in Figures 15, 16, and 17 confirm that bursting synchronization is observed under appropriate coupling intensity, while transition from synchronization to desynchronization occurs by further increasing the coupling intensity; interestingly, bursting synchronization emerges by greatly increasing the coupling intensity. That is to say, the bursting synchronization has no nothing with regular relation with the selection of coupling intensity in the case of bursting state for each neuronal circuit.

Extensive experimental results are practiced in the PSpice and the developed states of coupled neuronal circuits are detected to discern the transition of coupled neuronal circuits, and some results could be presented as follows. When each neuron is driven by external forcing current $I_{\text {ext }}=$ $120 \mu \mathrm{A}$, each neuron keeps quiescent state and quiescent synchronization is realized under coupling intensity $D=0.17$, 1.02 no matter whether nonreversing (or unidirectional) or bidirectional coupling being used. Spiking state is generated at $I_{\text {ext }}=130 \mu \mathrm{A}$, and spiking synchronization is realized by nonreversing (or unidirectional) coupling at coupling intensity $D=0.17,0.34$, while quiescent synchronization is realized by nonreversing (or unidirectional) coupling at coupling intensity $D=0.42,0.51,0.68,0.85,1.02$, and 1.1, where the potential mechanism could be that electrical activities of neurons are suppressed by stronger coupling. Spiking synchronization also makes bidirectional coupling at coupling intensity $D=0.17,0.34$, and 0.42 , and quiescent synchronization emerges at coupling intensity $D=0.51$, $0.68,0.85,1.02$, and 1.1. By further increasing the external forcing current as $I_{\text {ext }}=150 \mu \mathrm{A}$, thus bursting state and synchronization could be observed, and it is found that bursting synchronization occurs under nonreversing (or unidirectional) coupling at coupling intensity $D=0.17,0.34$, $0.51,0.68$, and 0.85 , while desynchronization begins to be observed at $D=1.02,1.1$. In the case of bidirectional coupling, bursting synchronization occurs at $D=0.17,0.34$, and 1.1, while desynchronization emerges at $D=0.51,0.68,0.85$, and 1.02. These results indicate that the final collective behavior (synchronization or desynchronization) could depend on the external forcing, coupling type, and also the coupling intensity as well, particularly when the neuron is in bursting state.

Above all, the evolution of coupled neuronal circuits is investigated by using PSpice tool, and it is found that the designed circuits are effective in detecting the development and transition of collective electric activities of neuronal network; indeed, the experimental results are much consistent with previous numerical studies and bifurcation analysis of neuronal activities. As a result, the designed neuronal circuit can be used as basic unit to study collective behavior of neuronal network with larger size and other potential applications. To be mentioned finally, it is thought that analog circuit by using commercial elements [39] or CMOL [40] and simulation program with integrated circuit emphasis (SPice) $[41,42]$ could be reliable to design intelligent circuit for signal process and analysis, and the synchronization between circuits could be useful for further study about collective behaviors of a large number of neuronal circuits. Indeed, these results based on simulation tools should be further improved in realistic experiments, similar to the mentioned experimental investigations [43-45] by generating multiscroll attractors or multiwings in chaotic circuit, FPGA, and integrated circuit with lower power consumption should be considered.

\section{Conclusions}

By using PSpice tool, a reliable and feasible neuronal circuit is designed, and the output analog signals for membrane potential are converted into digital signals via Schmitt trigger circuit; thus, the rhythm of output series could be detected, and the coupling feedback is realized by using subtracting 


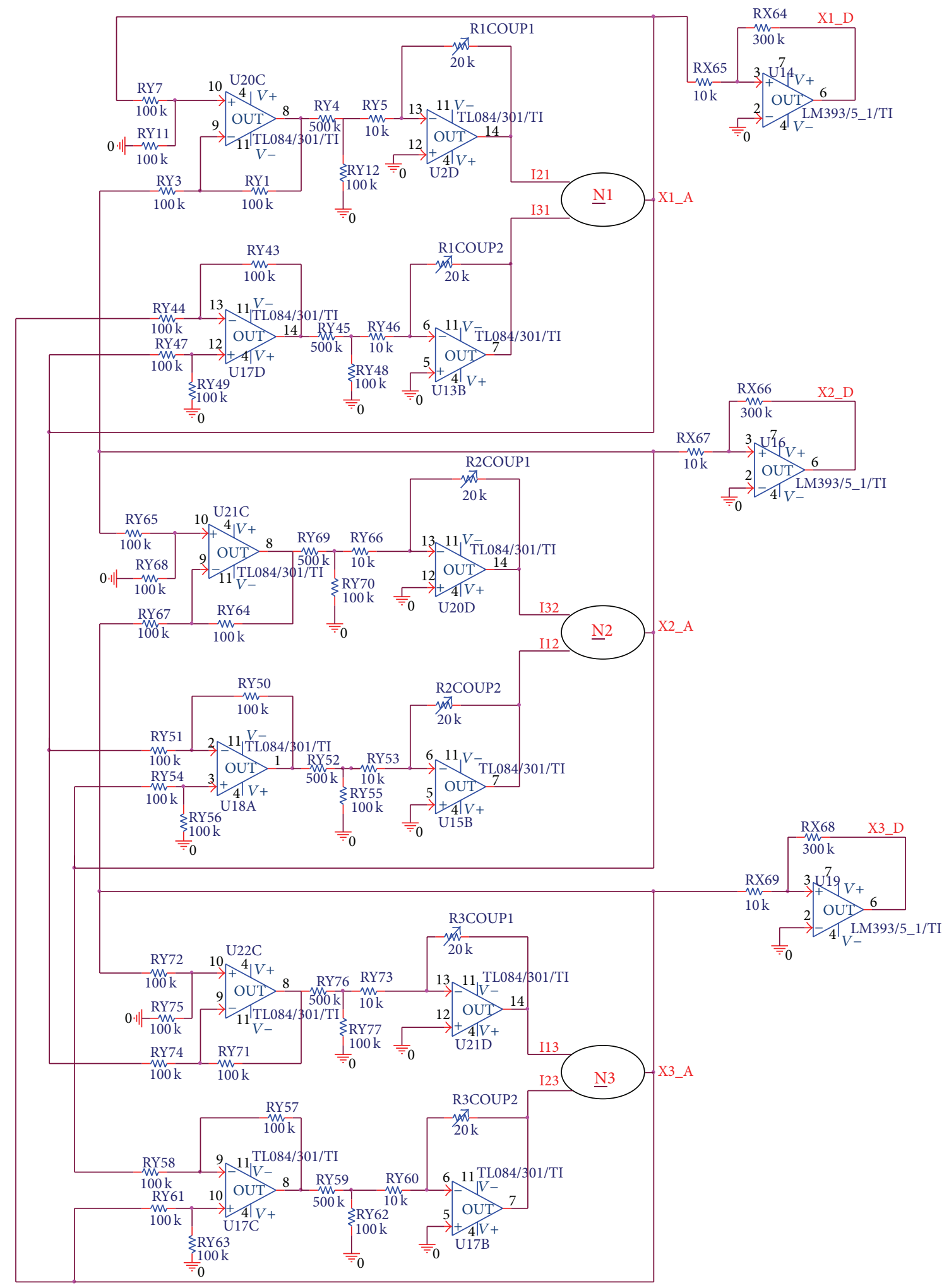

FIGURE 12: The coupled neuronal circuits for three neurons under bidirectional coupling type: X1_D, X2_D, and X3_D are the output digital signals from three different neuronal circuits; I31, I12, and I23 describe the coupling feedback on the first, second, and third neurons, respectively. 

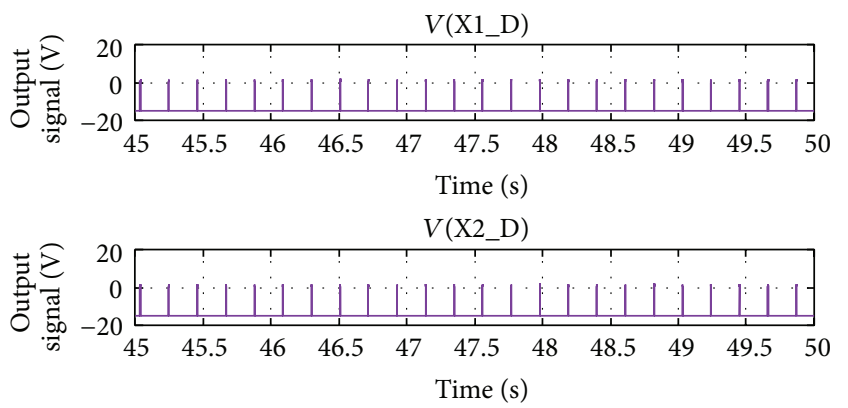

$V\left(\mathrm{X} 3 \_\mathrm{D}\right)$

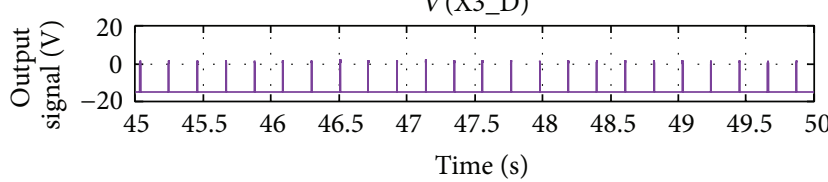

Figure 13: The digital outputs for three circuits via Schmitt trigger circuit: $I_{\text {ext }}=130 \mu \mathrm{A}$ and coupling intensity 0.17 is selected for bidirectional coupled neuronal circuit.
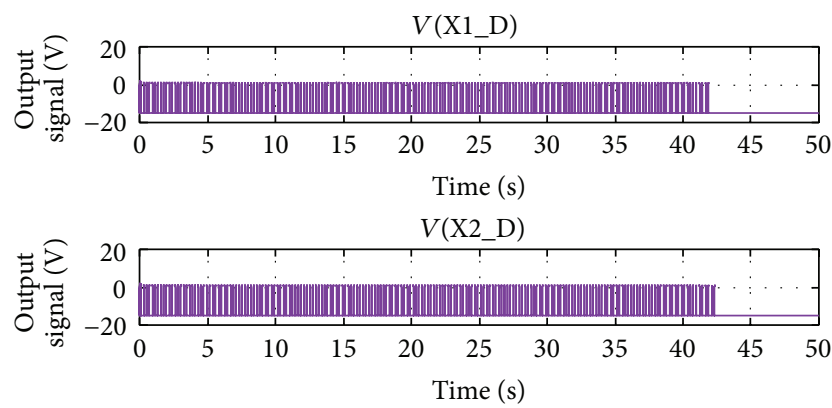

$V\left(\mathrm{X} 3 \_\mathrm{D}\right)$

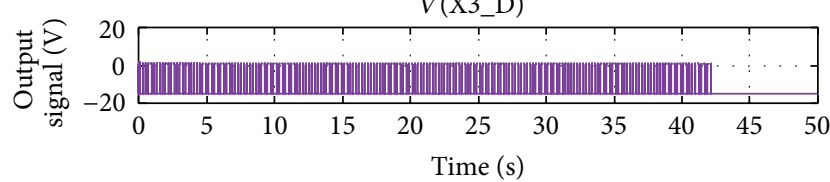

FIGURE 14: The digital outputs for three circuits via Schmitt trigger circuit: $I_{\text {ext }}=130 \mu \mathrm{A}$ and coupling intensity 0.5 is selected for bidirectional coupled neuronal circuit.

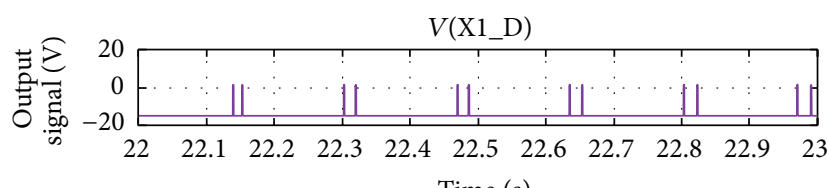

Time (s)

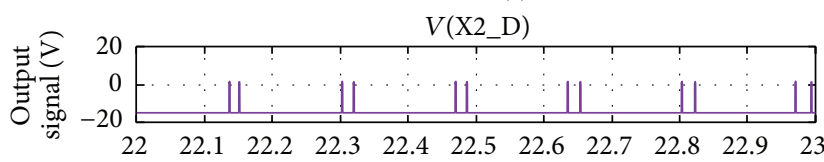

Time (s)

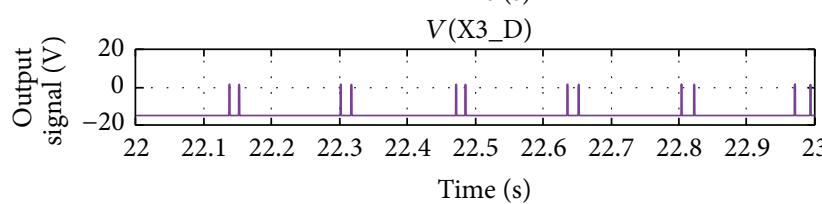

FIgURE 15: The digital outputs for three circuits via Schmitt trigger circuit: $I_{\text {ext }}=150 \mu \mathrm{A}$ and coupling intensity 0.34 is selected for bidirectional coupled neuronal circuit.

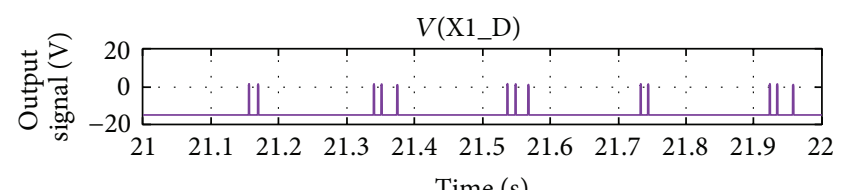

Time (s)

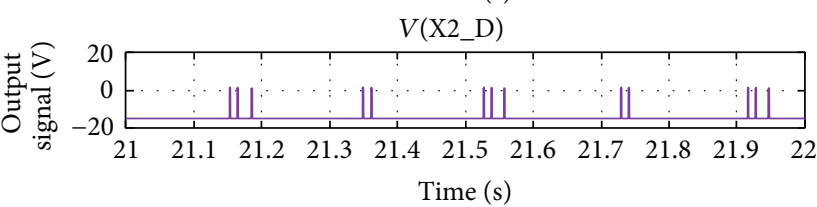

Time (s)

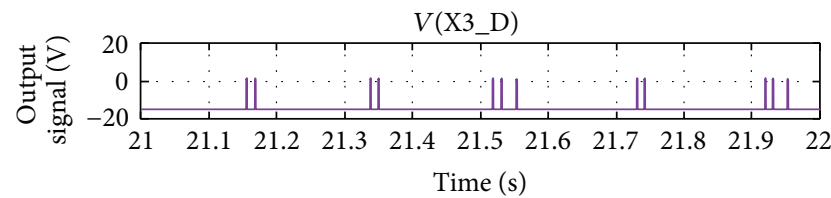

Figure 16: The digital outputs for three circuits via Schmitt trigger circuit: $I_{\text {ext }}=150 \mu \mathrm{A}$ and coupling intensity 0.5 is selected for bidirectional coupled neuronal circuit.

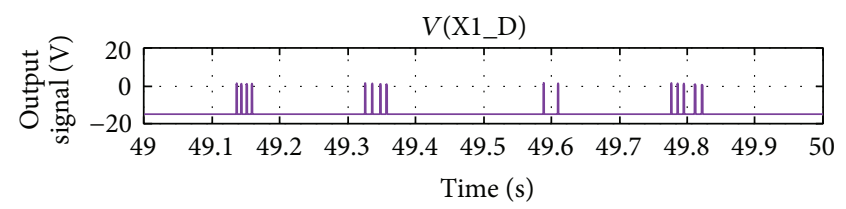

$V(\mathrm{X} 2 \mathrm{D})$

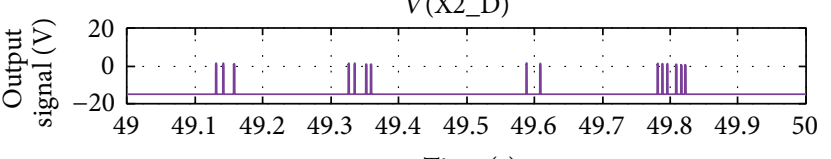

Time (s)

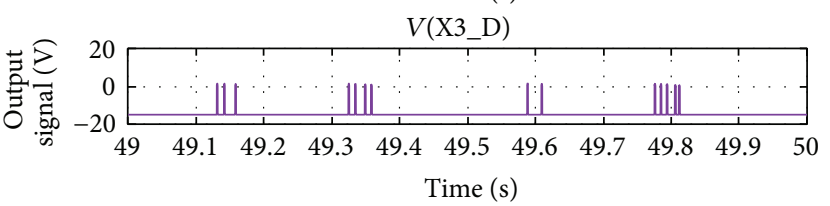

FIgURE 17: The digital outputs for three circuits via Schmitt trigger circuit: $I_{\text {ext }}=150 \mu \mathrm{A}$ and coupling intensity 1.01 is selected for bidirectional coupled neuronal circuit.

circuit based on Operational Amplifier TL08X. Furthermore, three neuronal circuits are connected to construct a network of coupled neuronal circuits; appropriate external forcing currents are applied to induce spiking, bursting, and quiescent state, respectively. The network is connected under nonreversing (or unidirectional) and bidirectional coupling type. It is found that appropriate coupling intensity enhances spiking synchronization while larger coupling intensity induces homogeneous state after spiking synchronization of the coupled neuronal circuits. In the case of bursting synchronization, transition from rhythm synchronization to desynchronization occurs alternatively by increasing the coupling intensity carefully. Both the nonreversing (or unidirectional) and bidirectional coupling connections between neuronal circuits can reproduce collective electric activities of neuronal network which can be adjusted by changing the external forcing current on neuronal circuit. And the designed network of coupled neuronal circuits is helpful to 
detect the transition of synchronization induced by other bifurcation parameters of the network.

\section{Competing Interests}

The authors declare that they have no competing interests.

\section{Acknowledgments}

This project is supported by the National Natural Science of Foundation of China (Grant no. 11365014) and the Gansu National Science of Foundation under Grant no. 1506RJZA095. And the authors would like to give great thanks to Dr. Ma J. (Associate Editor for Nonlinear Dynamics and also Academic Editor for PLoS One) for useful discussions.

\section{References}

[1] A. L. Hodgkin and A. F. Huxley, "A quantitative description of membrane current and its application to conduction and excitation in nerve," The Journal of Physiology, vol. 117, no. 4, pp. 500-544, 1952.

[2] J. L. Hindmarsh and R. M. Rose, "A model of neuronal bursting using three coupled first order differential equations," Proceedings of the Royal Society of London. Series B. Biological Sciences, vol. 221, no. 1222, pp. 87-102, 1984.

[3] B. Ibarz, J. M. Casado, and M. A. F. Sanjuán, "Map-based models in neuronal dynamics," Physics Reports, vol. 501, no. 1-2, pp. 174, 2011.

[4] J. Zhang, C. Wang, M. Wang, and S. Huang, "Firing patterns transition induced by system size in coupled Hindmarsh-Rose neural system," Neurocomputing, vol. 74, no. 17, pp. 2961-2966, 2011.

[5] Y. G. Yu, W. Wang, J. F. Wang, and F. Liu, "Resonanceenhanced signal detection and transduction in the HodgkinHuxley neuronal systems," Physical Review E, vol. 63, no. 2, pp. $1-12,2001$.

[6] J. Y. Hua and S. J. Smith, "Neural activity and the dynamics of central nervous system development," Nature Neuroscience, vol. 7, no. 4, pp. 327-332, 2004.

[7] R. B. Wang, Z. K. Zhang, and G. Chen, "Energy coding and energy functions for local activities of the brain," Neurocomputing, vol. 73, no. 1-3, pp. 139-150, 2009.

[8] J. Zhang, C. Shen, and Z. Cui, "Modulation on the collective response behavior by the system size in two-dimensional coupled cell systems," Science in China, Series G: Physics Astronomy, vol. 49, no. 3, pp. 304-312, 2006.

[9] Z. Wang, Y. Jiang, and H. Li, "Synchronization of multiple bursting neurons ring coupled via impulsive variables," Complexity, vol. 21, no. 2, pp. 29-37, 2015.

[10] Z.-L. Wang and X.-R. Shi, "Chaos bursting synchronization of mismatched Hindmarsh-Rose systems via a single adaptive feedback controller," Nonlinear Dynamics, vol. 67, no. 3, pp. 1817-1823, 2012.

[11] J. Fell and N. Axmacher, "The role of phase synchronization in memory processes," Nature Reviews Neuroscience, vol. 12, no. 2 , pp. 105-118, 2011.

[12] J.-W. Shuai and D. M. Durand, "Phase synchronization in two coupled chaotic neurons," Physics Letters A, vol. 264, no. 4, pp. 289-297, 1999.
[13] Q. Wang, H. Zhang, M. Perc, and G. Chen, "Multiple firing coherence resonances in excitatory and inhibitory coupled neurons," Communications in Nonlinear Science and Numerical Simulation, vol. 17, no. 10, pp. 3979-3988, 2012.

[14] Y. Wang, D. T. W. Chik, and Z. D. Wang, "Coherence resonance and noise-induced synchronization in globally coupled Hodgkin-Huxley neurons," Physical Review E, vol. 61, no. 1, pp. 740-746, 2000.

[15] D. Q. Wei, X. S. Luo, and Y. L. Zou, "Firing activity of complex space-clamped FitzHugh-Nagumo neural networks," European Physical Journal B, vol. 63, no. 2, pp. 279-282, 2008.

[16] M. Perc, "Spatial coherence resonance in excitable media," Physical Review E, vol. 72, no. 1, Article ID 016207, 6 pages, 2005.

[17] L. Wang, Y. B. Gong, X. Lin, and B. Xu, "Multiple coherence resonances by time-periodic coupling strength in scale-free networks of bursting neurons," European Physical Journal B, vol. 85, no. 1, article 14, 2012.

[18] M. Perc, "Spatial coherence resonance in neuronal media with discrete local dynamics," Chaos, Solitons \& Fractals, vol. 31, no. 1, pp. 64-69, 2007.

[19] D.-S. Lee, "Synchronization transition in scale-free networks: clusters of synchrony," Physical Review E, vol. 72, no. 2, Article ID 026208, 2005.

[20] H. X. Wang, Q. S. Lu, and Q. Y. Wang, "Bursting and synchronization transition in the coupled modified ML neurons," Communications in Nonlinear Science and Numerical Simulation, vol. 13, no. 8, pp. 1668-1675, 2008.

[21] B. Jia, "Synchronization transition of a coupled system composed of neurons with coexisting behaviors near a Hopf bifurcation," Chinese Physics B, vol. 23, no. 5, Article ID 050510, 2014.

[22] D. Q. Guo, Q. Y. Wang, and M. Perc, "Complex synchronous behavior in interneuronal networks with delayed inhibitory and fast electrical synapses," Physical Review E, vol. 85, no. 6, Article ID 061905, 8 pages, 2012.

[23] H. H. Zhang, Q. Y. Wang, M. Perc, and G. Chen, "Synaptic plasticity induced transition of spike propagation in neuronal networks," Communications in Nonlinear Science and Numerical Simulation, vol. 18, no. 3, pp. 601-615, 2013.

[24] H. Qin, J. Ma, W. Jin, and C. Wang, "Dynamics of electric activities in neuron and neurons of network induced by autapses," Science China Technological Sciences, vol. 57, no. 5, pp. 936-946, 2014.

[25] H. Qin, J. Ma, C. Wang, and R. Chu, "Autapse-induced target wave, spiral wave in regular network of neurons," Science China: Physics, Mechanics and Astronomy, vol. 57, no. 10, pp. 1918-1926, 2014.

[26] J. Ma, C. N. Wang, H. P. Ying, Y. Wu, and R. T. Chu, "Emergence of target waves in neuronal networks due to diverse forcing currents," Science China: Physics, Mechanics and Astronomy, vol. 56, no. 6, pp. 1126-1138, 2013.

[27] J. Ma, B. L. Hu, C. N. Wang, and W. Y. Jin, "Simulating the formation of spiral wave in the neuronal system," Nonlinear Dynamics, vol. 73, no. 1-2, pp. 73-83, 2013.

[28] X. Y. Wu, J. Ma, F. Li, and Y. Jia, "Development of spiral wave in a regular network of excitatory neurons due to stochastic poisoning of ion channels," Communications in Nonlinear Science and Numerical Simulation, vol. 18, no. 12, pp. 3350-3364, 2013.

[29] H. X. Qin, J. Ma, C. N. Wang, and Y. Wu, "Autapse-induced spiral wave in network of neurons under noise," PLOS ONE, vol. 9, no. 6, Article ID e100849, 2014. 
[30] H. G. Gu, B. B. Pan, G. R. Chen, and L. X. Duan, "Biological experimental demonstration of bifurcations from bursting to spiking predicted by theoretical models," Nonlinear Dynamics, vol. 78, no. 1, pp. 391-407, 2014.

[31] H. Gu and S. Chen, "Potassium-induced bifurcations and chaos of firing patterns observed from biological experiment on a neural pacemaker," Science China Technological Sciences, vol. 57, no. 5, pp. 864-871, 2014.

[32] H. G. Gu, "Biological experimental observations of an unnoticed chaos as simulated by the Hindmarsh-Rose model," PLoS ONE, vol. 8, no. 12, Article ID e81759, 2013.

[33] X. Y. Wu, J. Ma, L. H. Yuan, and Y. Liu, "Simulating electric activities of neurons by using PSPICE," Nonlinear Dynamics, vol. 75, no. 1-2, pp. 113-126, 2014.

[34] G.-D. Ren, G. Wu, J. Ma, and Y. Chen, "Simulation of electric activity of neuron by setting up a reliable neuronal circuit driven by electric autapse," Acta Physica Sinica, vol. 64, no. 5, Article ID 058702, 2015.

[35] E. Tamaševičiūtė, G. Mykolaitis, and A. Tamaševičius, "Analogue modelling an array of the FitzHugh-Nagumo oscillators," Nonlinear Analysis: Modelling and Control, vol. 17, pp. 118-125, 2012.

[36] J. Aliaga, N. Busca, V. Minces et al., "Electronic neuron within a ganglion of a leech (Hirudo medicinalis)," Physical Review E, vol. 67, no. 6, Article ID 061915, 2003.

[37] J. Ma, X. Y. Wu, R. T. Chu, and L. Zhang, "Selection of multiscroll attractors in Jerk circuits and their verification using Pspice," Nonlinear Dynamics, vol. 76, no. 4, pp. 1951-1962, 2014.

[38] K. Ding and Q.-L. Han, "Master-slave synchronization criteria for chaotic hindmarsh-roseneurons using linear feedback control," Complexity, 2015.

[39] A. Afifi, A. Ayatollahi, and F. Raissi, "CMOL implementation of spiking neurons and spike-timing dependent plasticity," International Journal of Circuit Theory and Applications, vol. 39, no. 4, pp. 357-372, 2011.

[40] C. Zhang and S. Yu, "On constructing complex grid multi-wing hyperchaotic system: theoretical design and circuit implementation," International Journal of Circuit Theory and Applications, vol. 41, no. 3, pp. 221-237, 2013.

[41] K. Yamamura and M. Tonokura, "Formulating hybrid equations and state equations for nonlinear circuits using SPICE," International Journal of Circuit Theory and Applications, vol. 41, no. 1, pp. 101-110, 2013.

[42] I. Vourkas, A. Batsos, and G. C. Sirakoulis, "SPICE modeling of nonlinear memristive behavior," International Journal of Circuit Theory and Applications, vol. 43, no. 5, pp. 553-565, 2015.

[43] J. L. V. S. de la Vega and E. Tlelo-Cuautle, "Simulation of piecewise-linear one-dimensional chaotic maps by Verilog-A," IETE Technical Review, vol. 32, no. 4, pp. 304-310, 2015.

[44] E. Tlelo-Cuautle, A. D. Pano-Azucena, V. H. Carbajal-Gomez, and M. Sanchez-Sanchez, "Experimental realization of a multiscroll chaotic oscillator with optimal maximum lyapunov exponent," The Scientific World Journal, vol. 2014, Article ID 303614, 16 pages, 2014.

[45] R. Trejo-Guerra, E. Tlelo-Cuautle, J. M. Jimenez-Fuentes et al., "Integrated circuit generating 3- and 5-scroll attractors," Communications in Nonlinear Science and Numerical Simulation, vol. 17, no. 11, pp. 4328-4335, 2012. 


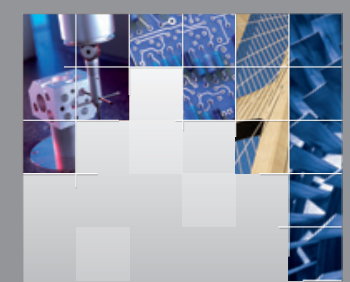

\section{Enfincering}
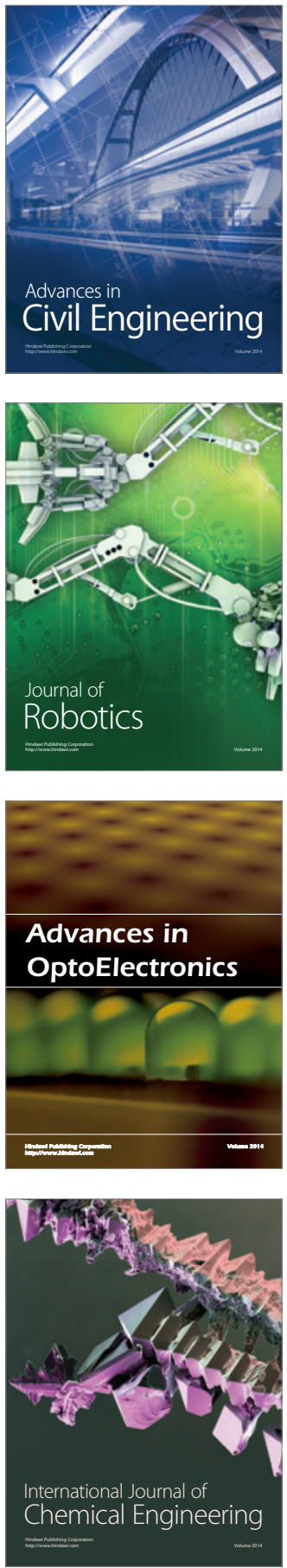

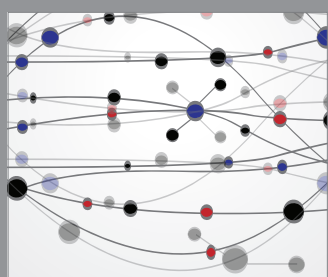

The Scientific World Journal

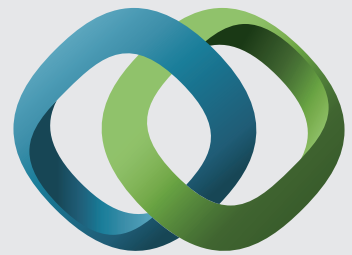

\section{Hindawi}

Submit your manuscripts at

http://www.hindawi.com
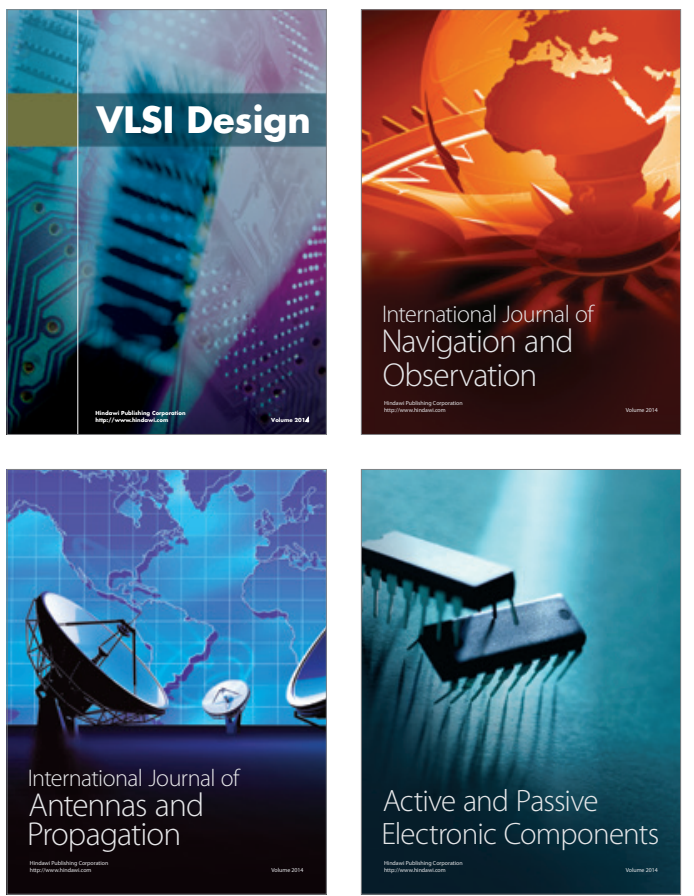
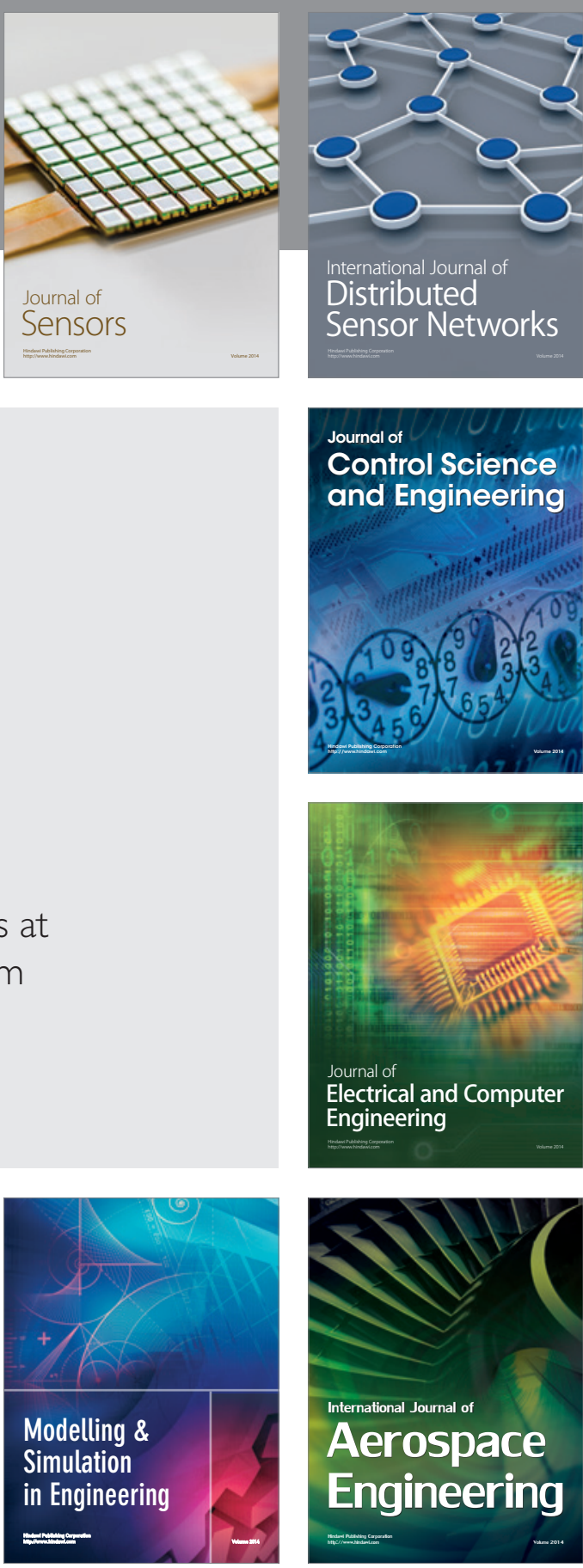

International Journal of

Distributed

Sensor Networks

Journal of

Control Science

and Engineering
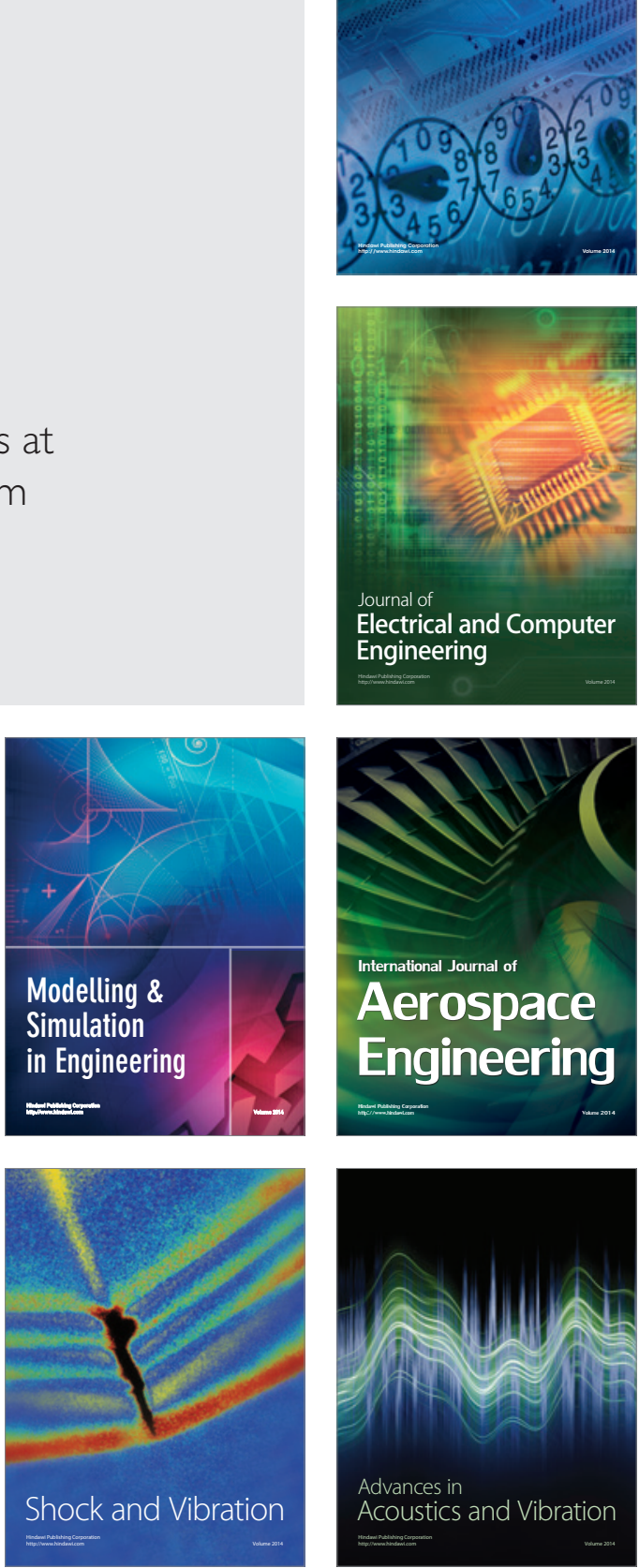